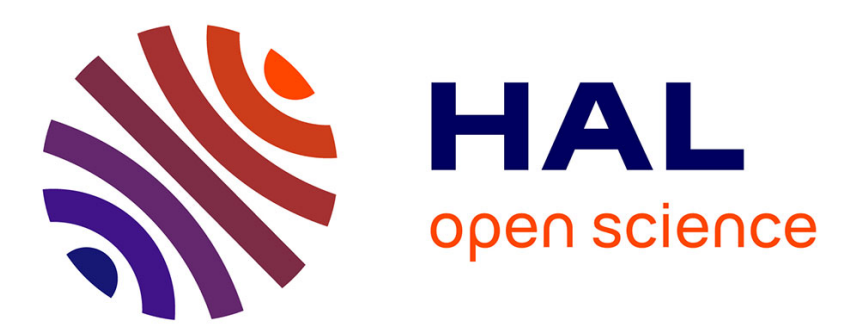

\title{
Stability Analysis of a More General Class of Systems with Delay-Dependent Coefficients
}

Chi Jin, Keqin Gu, Islam Boussaada, Silviu-Iulian Niculescu

\section{To cite this version:}

Chi Jin, Keqin Gu, Islam Boussaada, Silviu-Iulian Niculescu. Stability Analysis of a More General Class of Systems with Delay-Dependent Coefficients. IEEE Transactions on Automatic Control, 2019, 14, pp.1. 10.1109/TAC.2018.2832459 . hal-01957472

\section{HAL Id: hal-01957472 https://hal.science/hal-01957472}

Submitted on 17 Dec 2018

HAL is a multi-disciplinary open access archive for the deposit and dissemination of scientific research documents, whether they are published or not. The documents may come from teaching and research institutions in France or abroad, or from public or private research centers.
L'archive ouverte pluridisciplinaire $\mathbf{H A L}$, est destinée au dépôt et à la diffusion de documents scientifiques de niveau recherche, publiés ou non, émanant des établissements d'enseignement et de recherche français ou étrangers, des laboratoires publics ou privés. 


\title{
Stability Analysis of a More General Class of Systems with Delay-Dependent Coefficients
}

\author{
Chi Jin, Keqin Gu, IEEE Senior Member, Islam Boussaada, and Silviu-Iulian Niculescu, IEEE Fellow
}

\begin{abstract}
This paper presents a systematic method to analyse the stability of systems with single delay in which the coefficient polynomials of the characteristic equation depend on the delay. Such systems often arise in, for example, life science and engineering systems. A method to analyze such systems was presented by Beretta and Kuang in a 2002 paper, but with some very restrictive assumptions. This work extends their results to the general case with the exception of some degenerate cases. It is found that a much richer behavior is possible when the restrictive assumptions are removed. The interval of interest for the delay is partitioned into subintervals so that the magnitude condition generates a fixed number of frequencies as functions of the delay within each subinterval. The crossing conditions are expressed in a general form, and a simplified derivation for the first-order derivative criterion is obtained. Illustrative examples are also presented.
\end{abstract}

Index Terms-Delay Systems, Stability Analysis

\section{INTRODUCTION}

The presence of time-delay has been widely observed in physical and engineering systems, and it is often caused by the finite time needed to transfer materials, energy and information. Such systems may be modeled as delay deferential equations, and have attracted significant attentions of scholars from mathematics, engineering, life science and economics for many years. See [3], [9], [10], [11] for some recent progress.

For a linear system with constant coefficients and single delay or multiple commensurate delays, a number of effective methods have been proposed [1], [2], [5]. The methods are along the line of D-subdivision [6], [7], also known as the $\tau$-decomposition method [17] as the parameter involved in this case is the delay $\tau$. These methods roughly proceed as follows: starting with one value of delay $\tau_{0}$ that one knows the number of characteristic roots on the right-half plane (usually $\tau_{0}=0$ ), one sweeps through an interested interval $\left(\tau_{0}, \tau_{N}\right)$ of delays, and identify all delays $\tau_{k}, k=1,2, \ldots, N-1$ for which there are characteristic roots on the imaginary axis. By identifying the direction these roots cross the imaginary axis, the change of the number of right-half plane roots as $\tau$ goes through $\tau_{k}$ can be determined. Thus, the interval $\left(\tau_{0}, \tau_{N}\right)$ is divided into subintervals $\left(\tau_{k-1}, \tau_{k}\right)$, and the number of righthalf plane roots within each subinterval is constant and can

Chi Jin and Islam Boussaada are with IPSA \& Laboratoire des Signaux et Systèmes (L2S) CentraleSupélec-CNRS-Université Paris Sud, 3 rue JoliotCurie 91192 Gif-sur-Yvette cedex, France.

Keqin Gu is with Department of Mechanical and Industrial Engineering, Southern Illinois University Edwardsville, Edwardsville, Illinois 62026, USA.

Silviu-Iulian Niculescu is with Laboratoire des Signaux et Systèmes (L2S) CentraleSupélec-CNRS-Université Paris Sud, 3 rue Joliot-Curie 91192 Gifsur-Yvette cedex, France.

Manuscript received ??April 19, 2005; revised ??August 26, 2015. be explicitly determined. Especially, the subintervals of delay for the systems to be stable can be identified.

There are, however, practical systems in, for example, life science and engineering, for which the coefficients of the system characteristic equation depend on the delay values. For example, in [15], the source and dissipative process of a stellar dynamos is described by the following equations

$$
\left\{\begin{array}{l}
\dot{B}_{\phi}(t)=c_{1} e^{-c_{2} T_{0}} A\left(t-T_{0}\right)-c_{2} B_{\phi}(t), \\
\dot{A}(t)=c_{3} e^{-c_{2} T_{1}} B_{\phi}\left(t-T_{1}\right)-c_{2} A(t),
\end{array}\right.
$$

where $B_{\phi}$ is the strength of toroidal field, and $A$ is the strength of poloidal field, and $c_{1}, c_{2}, c_{3}, T_{0}, T_{1}$ are positive constants. The characteristic equation of the above system can be easily obtained as the following with delay-dependent coefficients:

$$
\lambda^{2}+2 c_{2} \lambda+c_{2}^{2}-c_{1} c_{3} e^{-c_{2} \tau} e^{-\tau \lambda}=0
$$

where $\tau=T_{0}+T_{1}$.

A model of hematopoietic stem cell dynamics is given in [16]. The model is nonlinear, and possesses two equilibria. The linearized equation in the neighborhood of the nonzero equilibrium has the following characteristic equation

$$
\lambda+A(\tau)-B(\tau) e^{-\lambda \tau}=0,
$$

where $A, B$ are nonlinear functions of $\tau$. Therefore delaydependent coefficients may result from the linearized dynamics of a nonlinear time-delay system.

Time-delay systems with delay-dependent coefficients can also arise from the analysis of partial differential equations. As an example, the modeling of cell density in a generic compartment in [27] suggests an advection or reaction-convection equation of the following form:

$$
\frac{\partial x(t, a)}{\partial t}+V \frac{\partial x(t, a)}{\partial a}=-\gamma(t, a) x(t, a) .
$$

A time-delay system can be obtained from the above equation using the method of characteristics

$$
\dot{S}(t)=2 \beta\left(S\left(t-\tau_{s}\right)\right) e^{-\gamma_{s} \tau_{s}} S\left(t-\tau_{s}\right)-[\beta(S(t))+\delta] S(t) .
$$

Detailed derivation and the meaning of the variables and functions in the above equation can be found in [27]. It is clear that the delay parameter $\tau$ enters the system coefficients through the exponential term $e^{-\gamma_{s} \tau_{s}}$.

Other examples of systems with delay-dependent coefficients include the sun flower model [26], control systems using a finite-difference scheme for stabilization [21] as well as various population dynamics models [14]. Indeed, it has been pointed out in [12] that the dynamics of a population that goes 
through distinct life stages in general involves delay-dependent parameters.

While it is possible to use the existing methods mentioned above to determine the stability of such a system with a given delay value, they are no longer sufficient to determine the range of delays for the system to be stable. Beretta and Kuang [12] presented an effective method to carry out such a stability analysis for systems with a single delay. However, the authors of [12] made some very restrictive assumptions, and the main attention has been paid to the crossing direction of the characteristic roots at the imaginary axis. No procedure was given in [12] to identify all the pairs $(j \omega, \tau)$ that satisfy the characteristic equation. In general, the structure of the functions $\omega(\tau)$ implicitly defined by $F(\omega, \tau)$ has not been sufficiently described in [12] to systematically identify all such pairs.

The purpose of this paper is to extend the method to the general case with the exception of some degenerate cases. As we will see, the removal of such restrictive assumptions means that a much richer behavior is possible. More specifically, the interval of interest for delay needs to be divided into subintervals so that the number of continuous functions $\omega(\tau)$ remains constant within each subinterval. The number of such functions may change as the delay moves from one subinterval to another. The dividing points of the interval are those delays for which two polynomial equations have a common real solution. Based on such a structure, the crossing delays and the corresponding crossing frequencies may be identified systematically. Furthermore, the delay intervals such that the system is stable may be determined based on the crossing directions of each critical delay-frequency pair.

The crossing direction in the general case may be determined numerically. With additional nondegeneracy assumption, the crossing direction may be conveniently determined analytically similar to the method given in [12], although we will show that a simplified derivation is possible.

A preliminary version of this paper was presented in [20].

The following notation will be used in this paper. For a polynomial, ord $(\cdot)$ denotes its order. For any complex number $c, \mathfrak{R}(c), \mathfrak{I}(c)$ and $\bar{c}$ denote its real part, imaginary part and conjugate, respectively. $\mathbb{R}$ stands for the set of real numbers and $\mathbb{R}_{+}$for non-negative reals. We will use $\partial$ with a subscript to denote partial derivatives. For instance, $\partial_{\lambda} D(\lambda, \tau):=\frac{\partial D(\lambda, \tau)}{\partial \lambda}$.

\section{Problem Statement}

Consider a time-delay system with characteristic equation of the form:

$$
D(\lambda, \tau)=P(\lambda, \tau)+Q(\lambda, \tau) e^{-\tau \lambda}=0,
$$

where $P(\lambda, \tau)$ and $Q(\lambda, \tau)$ are continuous in $\tau$ and are polynomials of $\lambda$ with real coefficients for each given $\tau \in \mathscr{I}$, and $\mathscr{I}=\left[\tau^{l}, \tau^{u}\right]$ is the range of delay parameters $\tau$ of interest, $0 \leq \tau^{l}<\tau^{u}$. In some context, we may write $P_{\tau}(\lambda)$ and $Q_{\tau}(\lambda)$ instead of $P(\lambda, \tau)$ and $Q(\lambda, \tau)$ in order to emphasize them as functions (polynomial in this case) of $\lambda$ for a given $\tau$. The same convention is also used for other functions of two independent variables with $\tau$ as one of them. For example, we may write $D_{\tau}(\lambda)$ instead of $D(\lambda, \tau)$ to emphasize that we are considering $D$ as a function of $\lambda$ for a given $\tau$ even though it is no longer a polynomial.

As we will see later on, the solutions of (2) with $\lambda$ on the imaginary axis plays an important role in stability analysis, in which case, (2) becomes

$$
D(j \omega, \tau)=0,
$$

where $\omega$ is real. For this purpose, we define:

$$
F(\omega, \tau)=P(j \omega, \tau) P(-j \omega, \tau)-Q(j \omega, \tau) Q(-j \omega, \tau) .
$$

It is not difficult to see that a necessary but not sufficient condition for $(\omega, \tau)$ to satisfy $(3)$ is

$$
F(\omega, \tau)=0 .
$$

The equation (5) is known as the magnitude condition, which means that the norms of the two complex number $P(j \omega, \tau)$ and $Q(j \omega, \tau)$ are equal.

We will restrict ourselves to systems that satisfy the following four assumptions:

Assumption I. For all $\tau \in \mathscr{I}, P_{\tau}$ satisfies

$$
\operatorname{ord}\left(P_{\tau}\right)=n
$$

Furthermore,

$$
\lim _{\omega \rightarrow \infty}\left|\frac{Q_{\tau}(j \omega)}{P_{\tau}(j \omega)}\right|<1 .
$$

Assumption II. No $(\omega, \tau) \in \mathbb{R}_{+} \times \mathscr{I}$ satisfies

$$
\begin{aligned}
& P(j \omega, \tau)=0, \\
& Q(j \omega, \tau)=0,
\end{aligned}
$$

simultaneously.

Assumption III. Any $\left(\omega^{*}, \tau^{*}\right) \in \mathbb{R}_{+} \times \mathscr{I}$ that satisfies (3) must also satisfy

$$
\left.\partial_{\lambda} D(\lambda, \tau)\right|_{\substack{\tau=\tau^{*} \\ \lambda=j \omega^{*}}} \neq 0
$$

Furthermore, let $\lambda(\tau)$ be the function implicitly defined by (2) in a sufficiently small neighborhood of $\left(j \omega^{*}, \tau^{*}\right)$ within $\mathbb{R}_{+} \times \mathscr{I}$, then for all $\tau \neq \tau^{*}, \tau \in \mathscr{I},\left|\tau-\tau^{*}\right|$ sufficiently small,

$$
\Re(\lambda(\tau)) \neq 0 .
$$

Assumption IV. There are only a finite number of $(j \omega, \tau)$ in $\mathbb{R}_{+} \times \mathscr{I}$ that simultaneously satisfy (5) and

$$
\partial_{\omega} F(\omega, \tau)=0 \text {. }
$$

These four assumptions are less restrictive than typical in the literature either stated explicitly or implicitly. Assumption I above requires the leading coefficient of $P_{\tau}$ not to vanish for all $\tau \in \mathscr{I}$, and

$$
\operatorname{ord}\left(Q_{\tau}\right) \leq n
$$

For time-delay systems of retarded type, (10) is satisfied with strict inequality. When (10) is an equality, the time-delay 
system is of neutral type, and (7) requires the absolute value of the leading coefficient of $Q_{\tau}(\lambda)$ to be strictly less than that of $P_{\tau}(\lambda)$. Systems of neutral type involve some surprising subtleties. See [2] for an example for systems with single delay. For more comprehensive coverage see [4] and [9].

Assumption II is much less restrictive than the counterpart in [12] which is

$$
P(j \omega, \tau)+Q(j \omega, \tau) \neq 0 \text { for all }(\omega, \tau) \in \mathbb{R}^{2} .
$$

Indeed, the two complex equations in Assumption II are equivalent to four real equations with two real "unknowns" $\omega$ and $\tau$. Obviously, cases that violate this assumption are degenerate and rare. On the other hand, the set

$$
\left\{P(j \omega, \tau)+Q(j \omega, \tau) \mid(\omega, \tau) \in \mathbb{R}^{2}\right\}
$$

is a region in the complex plane, and (11) requires this region not to include the origin, which is obviously much more restrictive. As will be presented later, the analysis is based on the phase condition on the set of parameters that satisfy the magnitude condition (5). The violation of this assumption makes the phase condition discontinuous at this point, and requires separate treatment which will not be pursued here.

In Assumption III, Condition (8) guarantees that $\lambda\left(\tau^{*}\right)=$ $j \omega^{*}$ is a simple characteristic root, and consequently $\lambda(\tau)$ is well defined in a small neighborhood of $\tau=\tau^{*}$ by the implicit function theorem [22], and $\lambda^{\prime}(\tau)$ exists at $\tau^{*}$ if $D(\lambda, \tau)$ is differentiable with respect to $\tau$ at $\left(\lambda\left(\tau^{*}\right), \tau^{*}\right)$. The remaining part of the assumption means that the curve $\lambda(\tau)$ is on the imaginary axis only at one point $\lambda^{*}=\lambda\left(\tau^{*}\right)$ in this neighborhood. A more restrictive assumption is to assume $\Re\left(\lambda^{\prime}(\tau)\right) \neq 0$, which is implicitly assumed in most works of this nature, including [12].

Assumption IV is also rather natural. It requires two real equations in two real variables to admit a finit number of solutions in the set $\mathbb{R}_{+} \times \mathscr{I}$. This assumption holds for most systems with delay dependent coefficients in practice. This assumption allows the delay interval $\mathscr{I}$ to be divided into a finite number of sub-intervals such that the polynomial $F_{\tau}(\omega)$ has a constant number of simple positive roots within each subinterval.

In most cases, we may choose the lower limit $\tau^{l}$ of $\mathscr{I}$ to be 0 , and the upper limit $\tau^{u}$ sufficiently large. We leave them in this general form so that the method we present here is still valid even if some of the assumptions are violated for some $\tau<\tau^{l}$ or $\tau>\tau^{u}$.

This paper provides extension of the analysis in [12] so that it is still applicable when the condition (11) is violated. In [12], it is also implicitly assumed that the number of real roots, $\pm \omega_{k}(\tau), k=1,2, \cdots, m$, of $F_{\tau}(\omega)$ remains constant within the delay interval of interest $\mathscr{I}$, and they are continuously differentiable. With our relaxed assumptions, these are no longer true. Especially, the real roots may suddenly emerge or disappear as the delay $\tau$ increases within $\mathscr{I}$. It is therefore essential to understand the structure of this solution set in order to solve the stability problem. This will be discussed in the next section.

\section{Stability ANALYSIS}

The main idea for stability analysis here is along the line of $\tau$-decomposition method outlined in the introduction. The validity of the method is based on the fact that there exists a constant $c>0$ for any closed interval of $\tau$ such that all roots of $D_{\tau}(\lambda)$ with $\Re(\lambda)>-c$ vary continuously as $\tau$ changes. This is true under Assumption I [2][4][9].

The critical aspects of the stability analysis are: (i) identifying the values of $\tau$ such that there is at least one root of $D_{\tau}(\lambda)$ on the imaginary axis, as well as the corresponding imaginary roots, and (ii) determining whether these imaginary roots move from the left-half plane to the right-half plane, or vise versa, or return to the original side as $\tau$ increases through these values. In this section, we will consider the first aspect, and describe the process of stability analysis assuming we know the answer to the second aspect. In the next section, we will describe some methods of accomplishing the second aspect.

To accomplish the first aspect stated in the last paragraph, it is useful to introduce the notation

$$
S(\lambda, \tau)=-\frac{P(\lambda, \tau)}{Q(\lambda, \tau)} e^{\tau \lambda}
$$

whenever

$$
Q(\lambda, \tau) \neq 0
$$

Then

$$
S(j \omega, \tau)=W(\omega, \tau) e^{j \theta(\omega, \tau)},
$$

where

$$
\begin{aligned}
W(\omega, \tau) & =\left|\frac{P(j \omega, \tau)}{Q(j \omega, \tau)}\right|, \\
\theta(\omega, \tau) & =\angle P(j \omega, \tau)-\angle Q(j \omega, \tau)+\omega \tau+\pi .
\end{aligned}
$$

When $\lambda=j \omega$ is on the imaginary axis, we note that (3) is equivalent to the following two conditions

$$
\begin{aligned}
W(\omega, \tau) & =1, \\
\theta(\omega, \tau) & =2 r \pi, \text { for some integer } r,
\end{aligned}
$$

provided that (13) holds.

Equation (17) is equivalent to (5), and represents the magnitude condition. Equation (18) is the phase condition. To capture essentially the same phase relationship, in [12] a function different from $\theta(\omega, \tau)$ is introduced, which requires the more restrictive condition (11). Let

$$
\mathscr{W}=\{(\tau, \omega) \mid \tau \in \mathscr{I}, \omega \in \mathbb{R}, F(\omega, \tau)=0\},
$$

then $(\tau, \omega) \in \mathscr{W}$ if and only if $(\tau, \omega)$ satisfies (13) and (17) in view of Assumption II. Therefore, an effective approach to determine all $(\tau, \omega)$ satisfying (3) is to first determine the set $\mathscr{W}$, and then choose from $\mathscr{W}$ those $(\tau, \omega)$ that also satisfy (18).

To understand the structure of $\mathscr{W}$, we will examine the function $F(\omega, \tau)=F_{\tau}(\omega)$ more closely. For any given $\tau$, $F_{\tau}(\omega)$ is an $2 n^{t h}$ order polynomial with real coefficients in 
view of Assumption I, and it is an even function. It can also be written as an $n^{\text {th }}$ order polynomial of $\omega^{2}$,

$$
\begin{aligned}
\hat{F}(\alpha, \tau) & =F(\omega, \tau), \\
\alpha & =\omega^{2} .
\end{aligned}
$$

Therefore

$$
\hat{F}(\alpha, \tau)=0
$$

will provide $n$ solutions $\alpha_{k}, k=1,2, \ldots, n$. Without loss of generality, let $\alpha_{k}, k=1,2, \ldots, n_{p}, n_{p} \leq n$, be the only real and non-negative solutions. Then, all the real solutions of (5) are $\pm \omega_{k}, k=1,2, \ldots, n_{p}$, where $\omega_{k}=\sqrt{\alpha_{k}}$. In general, the number of non-negative real roots $n_{p}$ depends on $\tau$. In order to understand this dependence, let $\tau^{(i)}, i=1,2, \ldots, K-1$ be the set of all $\tau \in \mathscr{I}$ such that $(\omega, \tau)$ simultaneously satisfies (5) and (9) for some $\omega \in \mathbb{R}_{+}$(recall this set is indeed finite according to Assumption IV). We agree to order $\tau^{(i)}$ in ascending order

$$
\tau^{(1)}<\tau^{(2)}<\cdots<\tau^{(K-1)} .
$$

We will also write $\tau^{(0)}=\tau^{l}$ and $\tau^{(K)}=\tau^{u}$. Then, we may partition $\mathscr{I}$ into $K$ subintervals

$$
\mathscr{I}^{(i)}=\left[\tau^{(i-1)}, \tau^{(i)}\right], i=1,2, \ldots, K .
$$

The interior of $\mathscr{I}^{(i)}$ is denoted as $\mathscr{I}_{o}^{(i)}=\left(\tau^{(i-1)}, \tau^{(i)}\right)$. Then the structure of the set $\mathscr{W}$ may be very clearly described in the following proposition.

Proposition 1. For a given $i$, the number of real roots of $F_{\tau}(\omega)$ are the same for all $\tau \in \mathscr{I}_{o}^{(i)}$, and they are all simple. These real simple roots are continuous functions of $\tau$, and may be expressed as $\pm \omega_{k}^{(i)}(\tau), k=1,2, \ldots, m^{(i)}$, where $m^{(i)} \leq n$, and $\omega_{k}^{(i)}(\tau)>0$ for all $\tau \in \mathscr{I}_{o}^{(i)}$.

Proof. For a fixed $i$, by definition, for all $\tau \in \mathscr{I}_{o}^{(i)}$, any $\omega \in \mathbb{R}$ that satisfies

$$
F_{\tau}(\omega)=0
$$

must satisfy

$$
F_{\tau}^{\prime}(\omega)=\partial_{\omega} F(\omega, \tau) \neq 0,
$$

from which we conclude that all real roots of $F_{\tau}(\omega)$ are simple. As $F_{\tau}(\omega)$ is an even function of $\omega$, we can also conclude that the $-\omega$ is also a root if $\omega$ is a real root, and $\omega=0$ is not a root (otherwise, it cannot be simple). To show the invariance of the number of real solutions within $\mathscr{I}_{o}^{(i)}$, let $\tau^{*} \in \mathscr{I}_{o}^{(i)}$, and let $\omega_{k}^{*}, k=1,2, \ldots, m$ be the only real roots of $F_{\tau^{*}}(\omega)$. By the continuity of roots with respect to coefficients[8], we may define $m$ continuous functions $\omega_{k}(\tau), k=1,2, \ldots, m$ in $\mathscr{I}_{o}^{(i)}, \omega_{k}\left(\tau^{*}\right)=\omega_{k}^{*}$, and each $\omega_{k}(\tau)$ is a root of $F_{\tau}(\omega)$. The proof is complete if we show that all $\omega_{k}(\tau)$ are real in $\mathscr{I}_{o}^{(i)}$ as this also implies that $\omega_{k}(\tau)$ are simple roots of $F_{\tau}(\omega)$.

For a given $k$, let

$$
\tau_{M}=\sup \left\{\tau_{a} \mid \omega_{k}(\tau) \in \mathbb{R} \text { for all } \tau \in\left[\tau^{*}, \tau_{a}\right]\right\} .
$$

By continuity, $\omega_{k}\left(\tau_{M}\right)$ is real. We will show $\tau_{M}=\tau^{(i)}$. If not, for arbitrarily small $\varepsilon>0, \omega_{k}\left(\tau_{M}+\varepsilon\right)$ is not real, which can be made arbitrarily close to $\omega_{k}\left(\tau_{M}\right)$ with sufficiently small $\varepsilon$. But this means that its complex conjugate $\bar{\omega}_{k}\left(\tau_{M}+\varepsilon\right)$ is also a root of the polynomial with real coefficients $F_{\tau_{M+\varepsilon}}(\omega)$ and arbitrarily close to $\omega_{k}\left(\tau_{M}\right)$. The continuity of roots with respect to the coefficients means that $\omega_{k}\left(\tau_{M}\right)$ cannot be a simple root of $F_{\tau_{M}}(\omega)$, which contradicts the first part of this proposition that we have already proven. We have thus shown that $\omega_{k}(\tau)$ is real for all $\tau \in\left[\tau^{*}, \tau^{(i)}\right)$. Similarly, we can show that $\omega_{k}(\tau)$ is real for all $\tau \in\left(\tau^{(i-1)}, \tau^{*}\right]$, and the proof is complete.

As $\tau$ moves rightward from a point in $\mathscr{I}_{o}^{(i)}$, some, say $m$, real roots, and $2 l$ complex roots of $F_{\tau}(\omega)$ may merge to form a multiple root as $\tau$ reaches $\tau^{(i)}$, and some, say $2 k$, become complex while $m+2 l-2 k$ roots remain real as $\tau$ enters $\mathscr{I}_{o}^{(i+1)}$. The most common scenarios are either two real roots merge and become complex, or two complex roots merge and become real as $\tau$ moves from $\mathscr{I}_{o}^{(i)}$ to $\mathscr{I}_{o}^{(i+1)}$ through $\tau^{(i)}$.

A real root of $F_{\tau}(\omega)$ in $\mathscr{I}_{o}^{(i)}$, say $\omega_{k}^{(i)}(\tau), k \leq m^{(i)}$, that does not merge with other roots at $\tau^{(i)}$ remains real, and becomes $\omega_{l}^{(i+1)}$ for some $l \leq m^{(i+1)}$ as $\tau$ moves from $\mathscr{I}_{o}^{(i)}$ to $\mathscr{I}_{o}^{(i+1)}$ through $\tau^{(i)}$.

For a given $i$ and $k$, as $\omega_{k}^{(i)}$ depends on $\tau$ continuously in $\mathscr{I}_{o}^{(i)}$, we will require $\angle P\left(j \omega_{k}^{(i)}(\tau), \tau\right)$ and $\angle Q\left(j \omega_{k}^{(i)}(\tau), \tau\right)$ to be continuous functions of $\tau$. This means that

$$
\theta_{k}^{(i)}(\tau)=\theta\left(\omega_{k}^{(i)}(\tau), \tau\right), k=1,2, \cdots, m^{(i)}
$$

are continuous functions of $\tau$ within $\mathscr{I}_{o}^{(i)}$, and will be known as the phase functions. On the other hand, this continuity requirement means that the values of $\angle P\left(j \omega_{k}^{(i)}(\tau), \tau\right)$, $\angle Q\left(j \omega_{k}^{(i)}(\tau), \tau\right)$ and $\theta_{k}^{(i)}(\tau)$ may not be restricted to any $2 \pi$ range. Furthermore, if $\omega_{k}^{(i)}(\tau)$ and $\omega_{l}^{(i)}(\tau)$ merge at, say, $\tau^{(i)}$, and we extend the definition of $\theta_{k}^{(i)}(\tau)$ and $\theta_{l}^{(i)}(\tau)$ to $\tau^{(i)}$ by continuity, then it is possible that

$$
\theta_{k}^{(i)}(\tau)-\theta_{l}^{(i)}(\tau)=2 \pi r,
$$

for some integer $r \neq 0$ even though

$$
\omega_{k}^{(i)}\left(\tau^{(i)}\right)=\omega_{l}^{(i)}\left(\tau^{(i)}\right)
$$

Going through each interval $\mathscr{I}^{(i)}$ and each curve $\omega_{k}^{(i)}(\tau)$, we may identify all $\tau=\tau_{l}$ such that

$$
\theta_{k}^{(i)}\left(\tau_{l}\right)=2 \pi r, r \text { integer, }
$$

for some $k$ if $\tau_{l} \in \mathscr{I}^{(i)}$. Notice, the ends of the intervals, $\tau^{(i)}$, $i=0,1, \cdots, K$ should also be included. We will order such $\tau_{l}$ in an ascending order

$$
\tau^{l} \leq \tau_{1}<\tau_{2}<\cdots<\tau_{L} \leq \tau^{u} .
$$

Each $\tau_{l}$ is known as a critical delay. For each given $\tau_{l}$, it is possible that more than one $k$ satisfies (28), and we denote the corresponding $\omega_{k}^{(i)}\left(\tau_{l}\right) \geq 0$ as $\omega_{l h}, h=1,2, \cdots, H_{l}$. Therefore, we can identify all the pairs $\left(\omega_{l h}, \tau_{l}\right), h=1,2, \cdots, H_{l} ; l=$ $1,2, \cdots, L$, that satisfy (3).

It should also be pointed out that it is possible that a simple root of $D_{\tau}(j \omega)$ may be a double root of $F_{\tau}(\omega)$. In other words, for some $\tau=\tau^{(i)}$, an $\omega$ that simultaneously satisfy (5) and (9) may satisfy (18) without violating Assumption III. Such 
points pose special difficulty in determining crossing direction as will be shown in the next section.

Now we will describe the representation of the second aspect we mentioned at the beginning of this section, i.e., the movement of the imaginary roots. For a given pair $\left(\omega_{l h}, \tau_{l}\right)$ that satisfies (3), a sufficiently small $\varepsilon>0$, and any $\tau \in\left(\tau_{l}, \tau_{l}+\varepsilon\right)$, there is a unique solution $\lambda_{l h}^{+}$of (2) in the neighborhood of $j \omega_{l h}$. Assumption III and continuity means that $\mathfrak{R}\left(\lambda_{l h}^{+}\right)$must be nonzero, and have the same sign for any $\tau \in\left(\tau_{l}, \tau_{l}+\varepsilon\right)$. Similarly, let $\lambda_{l h}^{-}$be the unique solution of (2) in the neighborhood of $j \omega_{l h}$ corresponding to a given $\tau \in\left(\tau_{l}-\varepsilon, \tau_{l}\right)$, then $\mathfrak{R}\left(\lambda_{l h}^{-}\right)$must have the same sign for all such $\tau$. We define

$$
\operatorname{Inc}\left(\omega_{l h}, \tau_{l}\right)=\frac{\operatorname{sgn}\left(\Re\left(\lambda_{l h}^{+}\right)\right)-\operatorname{sgn}\left(\Re\left(\lambda_{l h}^{-}\right)\right)}{2} .
$$

If $\operatorname{Inc}\left(\omega_{l h}, \tau_{l}\right)=1$, a root of $D_{\tau}(\lambda)$ moves from the left-half plane to the right-half plane crossing the imaginary axis at $j \omega_{l h}$ as $\tau$ increases from $\tau_{l}-\varepsilon$ to $\tau_{l}+\varepsilon$. On the other hand, if $\operatorname{Inc}\left(\omega_{l h}, \tau_{l}\right)=-1$, then the root moves from the right-half plane to the left-half plane as $\tau$ increases from $\tau_{l}-\varepsilon$ to $\tau_{l}+\varepsilon$. If $\operatorname{Inc}\left(\omega_{l h}, \tau_{l}\right)=0$, the root moves towards the imaginary axis, touching it at $j \omega_{l h}$, then return to the same half plane without crossing the imaginary axis. We also define

$$
\operatorname{Inc}\left(\tau_{l}\right)=2 \sum_{h=1}^{H_{l}} \operatorname{Inc}\left(\omega_{l h}, \tau_{l}\right) \text {. }
$$

Then, as $\tau$ increases from $\tau_{l}-\varepsilon$ to $\tau_{l}+\varepsilon$, there is a net increase of $\operatorname{Inc}\left(\tau_{l}\right)$ roots on the right-half plane. Notice, $\omega_{l h}>0, h=$ $1,2, \ldots, H_{l}$ only accounts for the roots on the upper half of the imaginary axis, and the coefficient 2 in front of the summation sign in (30) accounts for the fact that the roots of $D_{\tau}(\lambda)$ are symmetric to the real axis.

Let the number of right-half plane roots of $D_{\tau}(\lambda)$ be $N^{u}(\tau)$. Then, for any $\tau \in \mathscr{I}, \tau \neq \tau_{l}, l=1,2, \ldots, L$, we have

$$
N^{u}(\tau)=N^{u}\left(\tau^{l}\right)+\sum_{l=1}^{L_{\tau}} \operatorname{Inc}\left(\tau_{l}\right)
$$

where $L_{\tau}=\max \left\{l \mid \tau_{l}<\tau\right\}$.

If $\tau^{l}=0$, as $D_{\tau^{l}}(\lambda)$ is a polynomial, $N^{u}\left(\tau^{l}\right)$ is easily obtained. If $\tau^{l}>0, N^{u}\left(\tau^{l}\right)$ may be obtained by a method covered in [5] or [1] (but notice the correction [2]). If there are imaginary roots for $D_{\tau^{l}}(\lambda), N^{u}\left(\tau^{l}\right)$ should not count these imaginary roots, and $\operatorname{Inc}\left(\omega_{1 h}, \tau^{l}\right)$ should be defined as,

$$
\operatorname{Inc}\left(\omega_{1 h}, \tau^{l}\right)= \begin{cases}1, & \text { if } \operatorname{sgn}\left(\Re\left(\lambda_{1 h}^{+}\right)\right)=1, \\ 0, & \text { otherwise }\end{cases}
$$

instead. Obviously, $N^{u}(\tau)$ remains the same in the interval $\left(\tau_{l}, \tau_{l+1}\right)$ for any given $l$. The system is stable if $N^{u}(\tau)=0$.

\section{CROSSING DIRECTION CONDITIONS}

In the last section, a procedure of determining the range of $\tau$ in $\mathscr{I}$ such that $D_{\tau}(\lambda)$ is stable has been developed, provided a method of determining $\operatorname{Inc}\left(\omega_{l h}, \tau_{l}\right)$ is available. It is not difficult to determine $\operatorname{Inc}\left(\omega_{l h}, \tau_{l}\right)$ according to the definition if a numerical method is used. Indeed, as the solution $\left(j \omega_{l h}, \tau_{l}\right)$ is already known for $D(\lambda, \tau)$, the Newton-Raphson method may be used to find the unique solution in the neighborhood of $j \omega_{l h}$ when $\tau$ is very close to $\tau_{l}$ and $D(\lambda, \tau)$ is differentiable with respect to $\tau$ in a neighborhood of $\left(\omega_{l h}, \tau_{l}\right)$ [8]. In many cases, however, a simple analytical method can be used, which will be described as follows.

The simplest case is when

$$
\Re\left(\lambda_{l h}^{\prime}(\tau)\right)_{\tau=\tau_{l}} \neq 0,
$$

where, $\lambda_{l h}(\tau)$ is the implicit function defined by (2) in the neighborhood of $\left(j \omega_{l h}, \tau_{l}\right)$ provided that $\lambda_{l h}(\tau)$ is differentiable at $\tau_{l}$. This can be guaranteed by requiring $D(\lambda, \tau)$ to be differentiable w.r.t $\tau$ at $\left(j \omega_{l h}, \tau_{l}\right)$ [8]. Indeed, provided that (33) is satisfied, it is easy to see

$$
\operatorname{Inc}\left(\omega_{l h}, \tau_{l}\right)=\operatorname{sgn}\left(\Re\left(\lambda_{l h}^{\prime}\left(\tau_{l}\right)\right)\right),
$$

if $\tau_{l}>\tau^{l}$. On the other hand, if $\tau_{l}=\tau^{l}$, we have

$$
\operatorname{Inc}\left(\omega_{l h}, \tau_{l}\right)=\max \left\{0, \operatorname{sgn}\left(\Re\left(\lambda_{l h}^{\prime}\left(\tau_{l}\right)\right)\right)\right\} .
$$

If (33) is violated, and $D(\lambda, \tau)$ is differentiable to a sufficiently high order at $\left(j \omega_{l h}, \tau_{l}\right)$, then it follows from equation (8) in Assumption III and the implicit function theorem that the derivatives of $\lambda(\tau)$ exist up to a sufficiently high order at the point $\left(j \omega_{l h}, \tau_{l}\right)[8]$. Consequently we may express $\operatorname{Inc}\left(\omega_{l h}, \tau_{l}\right)$ using higher order derivatives. Suppose

$$
\begin{aligned}
& \Re\left(\frac{d^{k} \lambda(\tau)}{d \tau^{k}}\right)_{\tau=\tau_{l}}=0, k=1,2, \ldots, m-1, \\
& \Re\left(\frac{d^{m} \lambda(\tau)}{d \tau^{m}}\right)_{\tau=\tau_{l}} \neq 0 .
\end{aligned}
$$

Then, if $\tau_{l}>\tau^{l}$, then

$\operatorname{Inc}\left(\omega_{l h}, \tau_{l}\right)= \begin{cases}\operatorname{sgn}\left(\Re\left(\frac{d^{m} \lambda\left(\tau_{l}\right)}{d \tau^{m}}\right)\right), & \text { if } m \text { is odd } \\ 0, & \text { if } m \text { is even }\end{cases}$

If $\tau_{l}=\tau^{l}$, on the other hand, then

$$
\operatorname{Inc}\left(\omega_{l h}, \tau_{l}\right)=\max \left\{0, \operatorname{sgn}\left(\Re\left(\frac{d^{m} \lambda\left(\tau_{l}\right)}{d \tau^{m}}\right)\right)\right\} .
$$

If the condition (8) in Assumption III is violated for some imaginary characteristic root $\lambda=j \omega^{*}$, we are then faced with a characteristic root with multiplicity and cannot regard it as a locally differentiable function of $\tau$. In this case, the trajectory of characteristic roots parameterized by $\tau$ may have several branches passing through the point $j \omega^{*}$ on the imaginary axis. One may still determine the increment in the number of unstable roots based on these branches of curves, which can be locally characterized by the Newton-Puiseux series. Comprehensive analysis of this problem can be found in [23], [24] and [25]. An eigenvalue perturbation approach is taken in [23] and [24], which applies also to systems represented by the state-space matrices, whilst the analysis in [25] is based on the characteristic equations.

We will now give an explicit expression of $\operatorname{sgn}\left(\Re\left(\lambda_{l h}^{\prime}\left(\tau_{l}\right)\right)\right)$ and leave the high-order analysis to future work. The expression is similar to that given in [12], but our derivation here is more succinct. For this purpose, we henceforth replace Assumption III by the following one: 
Assumption IIIa. Any pair $\left(\omega^{*}, \tau^{*}\right) \in \mathbb{R} \times \mathscr{I}$ that satisfies (3) must also satisfy

$$
\partial_{\omega} F\left(\omega^{*}, \tau^{*}\right) \neq 0 .
$$

Furthermore, $D(\lambda, \tau)$ is differentiable with respect to $\tau$ in a neighborhood of $\left(j \omega^{*}, \tau^{*}\right)$.

The above assumption is stronger than the first part of Assumption III as indicated by the following Lemma.

Lemma 1. Any pair $\left(\omega^{*}, \tau^{*}\right)$ that satisfies Assumption IIIa must also satisfy (8).

Proof. At $\left(\omega^{*}, \tau^{*}\right)$

$$
\begin{aligned}
F & =\bar{P} P-\bar{Q} Q=0 \\
e^{-\tau \lambda} & =-P / Q=-\bar{Q} / \bar{P} \\
\partial_{\lambda} D & =\partial_{\lambda} P+\left(\partial_{\lambda} Q\right) e^{-\tau \lambda}-\tau Q e^{-\tau \lambda}=0
\end{aligned}
$$

Therefore,

$$
\begin{aligned}
\partial_{\omega} F & =2 \Re\left(j \bar{P} \partial_{\lambda} P-j \bar{Q} \partial_{\lambda} Q\right) \\
& =-2 \mathfrak{I}\left(\bar{P} \partial_{\lambda} P-\bar{Q} \partial_{\lambda} Q\right) \\
& =-2 \mathfrak{I}\left(\bar{P} \partial_{\lambda} P-\bar{P} \frac{\bar{Q}}{\bar{P}} \partial_{\lambda} Q+\tau \bar{P} P\right) \\
& =-2 \mathfrak{I}\left(\bar{P} \partial_{\lambda} P+\bar{P} e^{-\tau \lambda} \partial_{\lambda} Q-\tau \bar{P} Q e^{-\tau \lambda}\right) \\
& =-2 \mathfrak{I}\left(\bar{P} \partial_{\lambda} D\right) .
\end{aligned}
$$

The above indicates that $\partial_{\omega} F\left(\omega^{*}, \tau^{*}\right) \neq 0$ implies (8).

It should be pointed out that the converse is not necessarily true. Indeed, the proof above shows that $\partial_{\omega} F\left(\omega^{*}, \tau^{*}\right)=0$ only implies that $\partial_{\lambda} D\left(j \omega^{*}, \tau^{*}\right)$ is parallel to $P\left(j \omega^{*}, \tau^{*}\right)$, which does not necessarily mean $\partial_{\lambda} D\left(j \omega^{*}, \tau^{*}\right)=0$.

Proposition 2. Let $\left(\omega^{*}, \tau^{*}\right) \in \mathbb{R} \times \mathscr{I}$ satisfy (3) and Assumption IIIa. Then (2) defines $\lambda$ as a differentiable function of $\tau$ in a sufficiently small neighborhood of $\left(j \omega^{*}, \tau^{*}\right)$, and

$$
\begin{array}{r}
\operatorname{sgn}\left(\Re\left(\frac{d \lambda}{d \tau}\right)_{\tau=\tau^{*}}\right)=\operatorname{sgn}\left(\partial_{\omega} F(\omega, \tau)\right)_{\substack{\tau=\tau^{*} \\
\omega=\omega^{*}}} \\
\times \operatorname{sgn}\left(\frac{d_{F} \theta}{d \tau}\right)_{\substack{\tau=\tau^{*} \\
\omega=\omega^{*}}},
\end{array}
$$

where

$$
\frac{d_{F} \theta}{d \tau}=\partial_{\omega} \theta \frac{d_{F} \omega}{d \tau}+\partial_{\tau} \theta
$$

is the total derivative of $\theta(\omega, \tau)$ with respect to $\tau$ when $\omega$ is considered as a function of $\tau$ defined implicitly by (5) in a sufficiently small neighborhood of $\left(\omega^{*}, \tau^{*}\right)$, and $\frac{d_{F} \omega}{d \tau}$ is the derivative of the function $\omega(\tau)$ so defined.

Proof. Lemma 1 and Assumption IIIa indicate that $\partial_{\lambda} D(\lambda, \tau) \neq 0$ and $\partial_{\tau} D(\lambda, \tau)$ exists in a neighborhood of $\left(j \omega^{*}, \tau^{*}\right)$. Therefore, the equation (2), or equivalently

$$
S(\lambda, \tau)=1,
$$

defines $\lambda$ as a differentiable function of $\tau$ in a small neighborhood of $\tau^{*}$ in view of the implicit function theorem. A differentiation of (40) yields

$$
\partial_{\lambda} S \frac{d \lambda}{d \tau}+\partial_{\tau} S=0
$$

from which

$$
\frac{d \lambda}{d \tau}=-\partial_{\tau} S / \partial_{\lambda} S=-\partial_{\tau} S \overline{\left(\partial_{\lambda} S\right)} /\left|\partial_{\lambda} S\right|^{2} .
$$

But, at $\lambda=j \omega^{*}$,

$$
\begin{aligned}
\partial_{\lambda} S(\lambda, \tau) & =\frac{1}{j} \partial_{\omega} S(j \omega, \tau) \\
& =\frac{1}{j}\left[\left(\partial_{\omega} W\right) e^{j \theta}+j\left(\partial_{\omega} \theta\right) W e^{j \theta}\right] \\
& =-j \frac{1}{W} \partial_{\omega} W+\partial_{\omega} \theta .
\end{aligned}
$$

In the last step, (40) and (14) have been used. Similarly, we may obtain

$$
\partial_{\tau} S=\frac{1}{W} \partial_{\tau} W+j \partial_{\tau} \theta
$$

Therefore,

$$
\begin{aligned}
\operatorname{sgn}\left(\Re\left(\frac{d \lambda}{d \tau}\right)\right)= & -\operatorname{sgn}\left(\Re \left(\left(\frac{1}{W} \partial_{\tau} W+j \partial_{\tau} \theta\right)\right.\right. \\
& \left.\left.\times\left(\partial_{\omega} \theta+j \frac{1}{W} \partial_{\omega} W\right)\right)\right) \\
= & \operatorname{sgn}\left(\frac{\partial_{\omega} W \partial_{\tau} \theta-\partial_{\tau} W \partial_{\omega} \theta}{W}\right) .
\end{aligned}
$$

When $\omega$ is a function of $\tau$ defined implicitly by (5), or equivalently by (17), we have:

$$
\frac{d_{F} \omega}{d \tau}=-\partial_{\tau} W / \partial_{\omega} W=-\partial_{\tau} F / \partial_{\omega} F
$$

In view of $\left|Q\left(\omega^{*}, \tau^{*}\right)\right|=\left|P\left(\omega^{*}, \tau^{*}\right)\right|$, it is easy to show that

$$
\left.\frac{1}{W} \partial_{\omega} W\right|_{\substack{\tau=\tau^{*} \\ \omega=\omega^{*}}}=\left.\frac{1}{|P|^{2}} \partial_{\omega} F\right|_{\substack{\tau=\tau^{*} \\ \omega=\omega^{*}}} .
$$

A substitution of (41) by (42) and (43) yields

$$
\operatorname{sgn}\left(\Re\left(\frac{d \lambda}{d \tau}\right)\right)=\operatorname{sgn}\left(\frac{1}{|P|^{2}} \partial_{\omega} F\left(\frac{d_{F} \omega}{d \tau} \partial_{\omega} \theta+\partial_{\tau} \theta\right)\right),
$$

from which (39) can be easily derived.

We now make a useful observation about the first factor in (39).

Proposition 3. For any given $i$ and $k$, the quantity

$$
\operatorname{sgn}\left(\partial_{\omega} F(\omega, \tau)\right)_{\omega=\omega_{k}^{(i)}(\tau)}
$$

remains constant for all $\tau \in \mathscr{I}_{o}^{(i)}$.

Proof. Due to the continuity of $\partial_{\omega} F(\omega, \tau)$, in order for $\partial_{\omega} F\left(\omega_{k}^{(i)}(\tau), \tau\right)$ to change sign, it must first vanish, which violates the definition of $\mathscr{I}_{o}^{(i)}$.

The above proposition indicates that the first factor in (39) only needs to be checked once for each curve $\omega_{k}^{(i)}(\tau)$ within 
the interval $\mathscr{I}_{o}^{(i)}$. Next, we will provide an explicit expression for the second factor.

Proposition 4. If $(\omega, \tau)$ satisfies (3),

$$
\begin{aligned}
\frac{d_{F} \theta}{d \tau}= & \frac{1}{|P|^{2}}\left(P_{r} \frac{d_{F} P_{i}}{d \tau}-P_{i} \frac{d_{F} P_{r}}{d \tau}-Q_{r} \frac{d_{F} Q_{i}}{d \tau}+Q_{i} \frac{d_{F} Q_{r}}{d \tau}\right) \\
& +\tau \frac{d_{F} \omega}{d \tau}+\omega,
\end{aligned}
$$

where the subscripts $r$ and $i$ represent the real and imaginary part of the quantities, respectively, and the total derivatives may be calculated by

$$
\frac{d_{F} \phi}{d \tau}=\partial_{\omega} \phi \frac{d_{F} \omega}{d \tau}+\partial_{\tau} \phi
$$

where $\phi$ may be $P_{r}, P_{i}, Q_{r}$ or $Q_{i}$, and

$$
\frac{d_{F} \omega}{d \tau}=-\partial_{\tau} F / \partial_{\omega} F \text {. }
$$

Proof. Consider the identity

$$
S=W e^{j \theta}=-\frac{P e^{j \omega \tau}}{Q} .
$$

By taking total derivative with respect to $\tau$, with $\omega(\tau)$ implicitly defined by (5), and noticing

$$
W(\omega(\tau), \tau)=1 \text { for all } \tau,
$$

we obtain

$j \frac{d_{F} \theta}{d \tau} W e^{-j \theta}=-\frac{d_{F}}{d \tau}\left(\frac{P}{Q}\right) e^{j \omega \tau}-j\left(\tau \frac{d_{F} \omega}{d \tau}+\omega\right) \frac{P e^{j \omega \tau}}{Q}$.

Solving the above for $d_{F} \theta / d \tau$ and using (45), we obtain

$$
\frac{d_{F} \theta}{d \tau}=\frac{1}{j}\left(\frac{1}{P} \frac{d_{F} P}{d \tau}-\frac{1}{Q} \frac{d_{F} Q}{d \tau}\right)+\tau \frac{d_{F} \omega}{d \tau}+\omega .
$$

In view of $|P|^{2}=|Q|^{2}$, the expression in the parentheses in (46) can be written as

$$
\frac{1}{P} \frac{d_{F} P}{d \tau}-\frac{1}{Q} \frac{d_{F} Q}{d \tau}=\frac{\bar{P}}{P \bar{P}} \frac{d_{F} P}{d \tau}-\frac{\bar{Q}}{Q \bar{Q}} \frac{d_{F} Q}{d \tau}=\frac{\bar{P} \frac{d_{F} P}{d \tau}-\bar{Q} \frac{d_{F} Q}{d \tau}}{P \bar{P}} .
$$

A substitution of (46) by the above completes the proof.

While no explicit expression was given for $d_{F} \theta / d \tau$ in [12], an explicit expression of $S_{n}^{\prime}(\tau)$ in [12] could be obtained by going through the proof of Theorem 2.2 in [12]. Proposition 3 above can be considered as the consequence of Theorem 2.2 and Remark 2.2 in [12]. Indeed, It can be seen that $S_{n}(\tau)$ in Theorem 2.2 in [12] is equal to $(\theta(\tau)-2 n \pi) / \omega(\tau)$ here. Remark 2.2 in [12] indicates that the factor $\omega(\tau)$ does not affect the sign of the derivative at the crossing point.

It is interesting to apply the conclusions of Proposition 2 to the case of delay-independent coefficient polynomials, i.e., when $P(\lambda, \tau)$ and $Q(\lambda, \tau)$ are independent of $\tau$. In this case, $F(\omega, \tau)$ is independent of $\tau$, the curves $\omega_{k}^{(i)}(\tau)$ become constants, and $d_{F} \theta / d \tau=\omega=$ constant. As a result, the crossing direction given in (39) is independent of delay. This fact is well-known in the literature on single or commensurate delay systems with delay-independent coefficients, and have been stated either implicitly [5] or explicitly [19] as the invariance property.
More generally, for systems with delay-dependent coefficient polynomials discussed in this paper, we may still identify delay intervals where the crossing direction is invariant provided $P(\lambda, \tau)$ and $Q(\lambda, \tau)$ are continuously differentiable with respect to $\tau$. Indeed, for a given subinterval $\mathscr{I}_{o}^{(i)}=\left(\tau^{(i-1)}, \tau^{(i)}\right)$, and frequency curve $\omega_{k}^{(i)}(\tau)$, we may identify all the delay values $\tau_{k l}^{(i)}, l=1,2, \ldots, L-1, \tau^{(i-1)}<$ $\tau_{k 1}^{(i)}<\tau_{k 2}^{(i)}<\cdots<\tau_{k, L-1}^{(i)}<\tau^{(i)}$, such that $\left(d_{F} \theta / d \tau\right)_{\tau=\tau_{k l}^{(i)}}=0$. Let $\tau_{k 0}^{(i)}=\tau^{(i-1)}, \tau_{k, L}^{(i)}=\tau^{(i)}$. Then, we may conclude, by continuity, that the crossing direction at the curve $\omega_{k}^{(i)}(\tau)$ remains invariant for all $\tau \in\left(\tau_{k, l-1}^{(i)}, \tau_{k l}^{(i)}\right), l=1,2, \ldots, L$. Note that the intervals for invariant crossing direction $\left(\tau_{k, l-1}^{(i)}, \tau_{k l}^{(i)}\right)$ are different for different frequency curves in general.

\section{NUMERICAL EXAMPLES}

In this section, we present three examples to illustrate the method developed in this paper.

Example 1. We first consider the stellar dynamos model mentioned in the introduction. The system characteristic equation is given in (1). Therefore,

$$
\begin{aligned}
P(\lambda, \tau) & =\lambda^{2}+2 c_{2} \lambda+c_{2}^{2}, \\
Q(\lambda, \tau) & =-c_{1} c_{3} e^{-c_{2} \tau} .
\end{aligned}
$$

The parameters are set as: $c_{1}=-10, c_{2}=2, c_{3}=3$. We are concerned with the stability of the system for $\tau \in \mathscr{I}=[0,2]$. Since $\operatorname{ord}\left(P_{\tau}\right)=2$ and $\operatorname{ord}\left(Q_{\tau}\right)=0$, Assumption I holds. Assumption II requires the following two equations do not hold simultaneously for real $\omega$ and $\tau \in \mathscr{I}$ :

$$
\begin{aligned}
-\omega^{2}+2 j c_{2} \omega+c_{2}^{2} & =0, \\
-c_{1} c_{3} e^{-c_{2} \tau} & =0,
\end{aligned}
$$

which is obviously true. The other assumptions can be verified as we carry out the remaining analysis. The function $F$ in this case is

$$
F(\omega, \tau)=\omega^{4}+2 c_{2}^{2} \omega^{2}+c_{2}^{4}-c_{1}^{2} c_{3}^{2} e^{-2 c_{2} \tau} .
$$

Only one pair of parameters $(\omega, \tau)=\left(0, \tau^{(1)}\right)$ simultaneously satisfies (5) and (9), where

$$
\tau^{(1)}=-\frac{1}{2 c_{2}} \ln \left(\frac{c_{2}^{4}}{c_{1}^{2} c_{3}^{2}}\right) \approx 1.006 .
$$

Therefore, Assumption IV is satisfied. The interval $\mathscr{I}$ is thus partitioned into two subintervals $\mathscr{I}^{(1)}=\left[\tau^{(0)}, \tau^{(1)}\right], \mathscr{I}^{(2)}=$ $\left[\tau^{(1)}, \tau^{(2)}\right]$, where $\tau^{(0)}=0, \tau^{(2)}=2$. There is one positive real root $\omega_{1}^{(1)}(\tau)$ of $F_{\tau}(\omega)$ for $\tau \in\left(0, \tau^{(1)}\right)$. As $\tau$ reaches $\tau^{(1)}$, this solution merges with the negative solution $-\omega_{1}^{(1)}(\tau)$, and they become complex as $\tau$ enters $\mathscr{I}^{(2)}$, and $F_{\tau}(\omega)$ does not have any real solution for $\tau$ in $\mathscr{I}^{(2)}$. In this case, we have

$$
\omega_{1}^{(1)}(\tau)=\sqrt{\left|c_{1} c_{3}\right| e^{-c_{2} \tau}-c_{2}^{2}} .
$$

Corresponding to $\omega=\omega_{1}^{(1)}(\tau), \theta_{1}^{(1)}(\tau)$ defined in (26) is plotted against $\tau$ in the top diagram of Figure 1. It can be seen that the curve intersects the horizontal line $2 \pi$ at $\tau_{1} \approx 0.2748$ 
and $\tau_{2} \approx 0.5314$. Therefore, $H_{1}=1, \omega_{11}=\omega_{1}^{(1)}\left(\tau_{1}\right) \approx 3.6490$, and $H_{2}=1, \omega_{21}=\omega_{1}^{(1)}\left(\tau_{2}\right) \approx 2.5228$. Since both $\tau_{1}$ and $\tau_{2}$ are different from $\tau^{(1)}$, it is easy to verify that Assumption IIIa holds because (9) does not hold for each $\left(\omega_{l h}, \tau_{l}\right)$. Assumption III is further implied by Assumption IIIa.
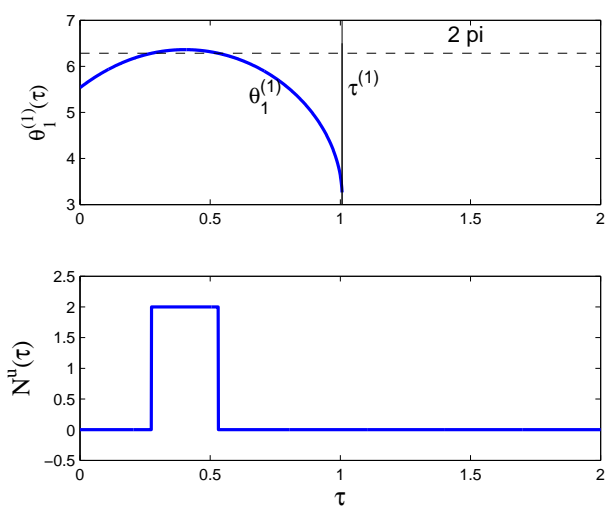

Fig. 1. The stability analysis of the stellar dynamos. The two intersections between the graph of $\theta_{1}^{(1)}$ and the black-dashed line located at $2 \pi$ corresponds to the two delay values for which the system has a pair of imaginary roots. $N^{u}$ is the number of unstable roots of the stellar dynamos.

It can be verified that $\partial_{\omega} F\left(\omega_{1}^{(1)}(\tau), \tau\right)>0$ for $\tau=0.5$, and the above inequality holds for all $\tau \in \mathscr{I}_{o}^{(1)}$ according to Proposition 3. It can be easily calculated that

$$
\frac{d}{d \tau} \theta_{1}^{(1)}\left(\tau_{1}\right)>0, \frac{d}{d \tau} \theta_{1}^{(1)}\left(\tau_{2}\right)<0,
$$

which are also obvious from the top diagram in Figure 1. Therefore, we conclude from (39) that a pair of characteristic roots cross the imaginary axis from the left-half plane to the right-half plane as $\tau$ increases through $\tau_{1}$, and this pair of characteristic roots return to the left-half plane as $\tau$ further increases through $\tau_{2}$. In other words, $\operatorname{Inc}\left(\omega_{11}, \tau_{1}\right)=1$, and $\operatorname{Inc}\left(\omega_{21}, \tau_{2}\right)=-1$. Some simple calculation shows that the system is asymptotically stable for $\tau=0$. A plot of $N^{u}(\tau)$ is shown in the bottom diagram of Figure 1, from which we conclude that the system is stable for $\tau \in\left[0, \tau_{1}\right) \cup\left(\tau_{2}, \tau^{(2)}\right]$; it is unstable for $\tau \in\left(\tau_{1}, \tau_{2}\right)$.

Example 2. Consider the following characteristic equation representing the population dynamics in [13],

$$
\lambda^{2}+a \lambda+c+(b(\tau) \lambda+d(\tau)) e^{-\lambda \tau}=0,
$$

where

$$
b(\tau)=k_{1} e^{-m \tau}, d(\tau)=k_{2} e^{-m \tau} .
$$

The parameters are set as:

$$
a=2, c=1, k_{1}=4, k_{2}=2, m=3.5 .
$$

We analyse the stability of the system for $\mathscr{I}=[0,2.5]$. By definiton, we have

$$
\begin{aligned}
& P(\lambda, \tau)=\lambda^{2}+a \lambda+c, \\
& Q(\lambda, \tau)=b(\tau) \lambda+d(\tau) .
\end{aligned}
$$

Since $\operatorname{ord}\left(P_{\tau}\right)=2$ and $\operatorname{ord}\left(Q_{\tau}\right)=1$, Assumption I holds. Assumption II requires the following two equations do not hold simultaneously for real $\omega$ and $\tau \in \mathscr{I}$ :

$$
\begin{aligned}
-\omega^{2}+a j \omega+c & =0, \\
b(\tau) j \omega+d(\tau) & =0,
\end{aligned}
$$

which can be easily verified to be true. The function $F$ in this case is

$$
F(\omega, \tau)=\omega^{4}+\left(a^{2}-b^{2}(\tau)-2 c\right) \omega^{2}+c^{2}-d^{2}(\tau) .
$$

Solving (5) and (9) together for $(\omega, \tau) \in \mathbb{R}_{+} \times \mathscr{I}$, we obtain two pairs of solutions approximately equal to $(0,1.981)$ and $(0.720,2.391)$. The interval $\mathscr{I}$ is thus partitioned into three subintervals $\mathscr{I}^{(1)}=\left[\tau^{(0)}, \tau^{(1)}\right], \mathscr{I}^{(2)}=\left[\tau^{(1)}, \tau^{(2)}\right], \mathscr{I}^{(3)}=$ $\left[\tau^{(2)}, \tau^{(3)}\right]$, where $\tau^{(0)}=0, \tau^{(1)} \approx 1.981, \tau^{(2)} \approx 2.391, \tau^{(3)}=$ 2.5. The polynomial $F_{\tau}(\omega)$ has one positive real root, namely $\omega_{1}^{(1)}(\tau)$, in the interval $\left(\tau^{(0)}, \tau^{(1)}\right)$ and two positive roots, namely $\omega_{1}^{(2)}(\tau)$ and $\omega_{2}^{(2)}(\tau)$, in the interval $\left(\tau^{(1)}, \tau^{(2)}\right)$. It has no real root for $\tau \in\left(\tau^{(2)}, \tau^{(3)}\right)$. We have the following expressions:

$$
\begin{aligned}
& \omega_{1}^{(1)}(\tau)=2^{-1 / 2} \sqrt{\left(b^{2}(\tau)+2 c-a^{2}\right)+\Delta^{1 / 2}(\tau)}, \tau \in \mathscr{I}^{(1)}, \\
& \omega_{1}^{(2)}(\tau)=2^{-1 / 2} \sqrt{\left(b^{2}(\tau)+2 c-a^{2}\right)+\Delta^{1 / 2}(\tau)}, \tau \in \mathscr{I}^{(2)}, \\
& \omega_{2}^{(2)}(\tau)=2^{-1 / 2} \sqrt{\left(b^{2}(\tau)+2 c-a^{2}\right)-\Delta^{1 / 2}(\tau)}, \tau \in \mathscr{I}^{(2)},
\end{aligned}
$$

where $\Delta(\tau)=\left(b^{2}(\tau)+2 c-a^{2}\right)^{2}-4\left(c^{2}-d^{2}(\tau)\right)$. We observe that $\pm \omega_{2}^{(2)}(\tau)$ emerge as a pair of real roots of $F_{\tau}(\omega)$ at $\tau=\tau^{(1)}$ and $\omega_{2}^{(2)}\left(\tau^{(1)}\right)=0$. As $\tau$ approaches $\tau^{(2)}$ from the left, the solution $\omega_{1}^{(2)}(\tau)$ merges with $\omega_{2}^{(2)}(\tau)$. These two positive roots become complex as $\tau$ increases beyond $\tau^{(2)}$. The corresponding phase functions $\theta_{1}^{(1)}(\tau), \theta_{1}^{(2)}(\tau), \theta_{2}^{(2)}(\tau)$ are plotted against $\tau$ in the top diagram of Figure 2. These curves intersect the horizontal line 0 at $\tau_{1} \approx 0.7576$ and $\tau_{2} \approx 2.1745$. Therefore, $H_{1}=1, \omega_{11}=\omega_{1}^{(1)}\left(\tau_{1}\right) \approx 2.7556$ and $H_{2}=1, \omega_{21}=\omega_{1}^{(2)}\left(\tau_{2}\right) \approx 1.1837$. Since both $\tau_{1}$ and $\tau_{2}$ are different from either $\tau^{(1)}$ or $\tau^{(2)}$, it is easy to see that (9) does not hold for each $\left(\omega_{l h}, \tau_{l}\right)$. Consequently we deduce that Assumption IIIa must hold, which also implies Assumption III.

It can be verified that

$$
\partial_{\omega} F\left(\omega_{1}^{(1)}(1), 1\right)>0, \partial_{\omega} F\left(\omega_{1}^{(2)}(2), 2\right)>0,
$$

therefore $\partial_{\omega} F\left(\omega_{1}^{(1)}(\tau), \tau\right)>0$ for $\tau \in\left(\tau^{(0)}, \tau^{(1)}\right)$ and $\partial_{\omega} F\left(\omega_{1}^{(2)}(\tau), \tau\right)>0$ for $\tau \in\left(\tau^{(1)}, \tau^{(2)}\right)$. Computation shows that

$$
\frac{d}{d \tau} \theta_{1}^{(1)}\left(\tau_{1}\right)>0, \frac{d}{d \tau} \theta_{1}^{(2)}\left(\tau_{2}\right)<0,
$$

which also follows from the graph of phase functions plotted in the top diagram of Fig.2. We deduce by using (39) that a pair of characteristic roots cross the imaginary axis from the left-half plane to the right-half plane as $\tau$ increases through $\tau_{1}$. Another pair of characteristic roots cross the imaginary axis from the right-half plane to the left-half plane as $\tau$ increases through $\tau_{2}$. Consequently, we have $\operatorname{Inc}\left(\omega_{11}, \tau_{1}\right)=1$ 

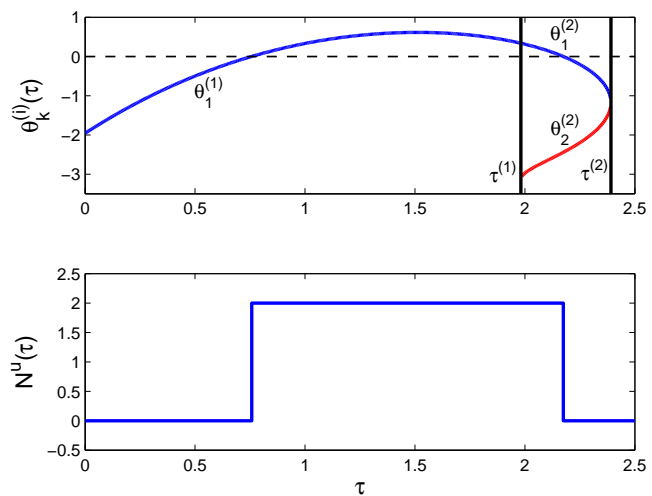

Fig. 2. The stability analysis of the population dynamics (48). The graphs of $\theta_{1}^{(1)}$ and $\theta_{1}^{(2)}$ intersect the black-dashed line located at 0 at $\tau_{1}$ and $\tau_{2}$ respectively, which correspond to the two delay values for which the system has a pair of imaginary characteristic roots. $N^{u}$ is the number of unstable roots of (48).

and $\operatorname{Inc}\left(\omega_{21}, \tau_{2}\right)=-1$. It is easy to verify that (48) is asymptotically stable for $\tau=0$. Therefore, we conclude that the system is asymptotically stable for $\tau \in\left[0, \tau_{1}\right) \cup\left(\tau_{2}, 2.5\right]$; it is unstable for $\tau \in\left(\tau_{1}, \tau_{2}\right)$. The plot of $N^{u}(\tau)$ is given in the bottom diagram of Figure 2.

In these two examples, after all the crossing pairs have been identified, the method in [12] may also be used to determine the crossing directions of each such pairs and thus completing the stability analysis. However, a systematic method to identify such pairs, which requires us to divide the delay interval of interest into sub-intervals, has not been considered in [12]. The following example shows that it is not always necessary to divide the interval of interest even if the condition (11), which is assumed in [12], is violated.

Example 3. Consider a system with the following characteristic equation for $\mathscr{I}=[0,1]$ :

$$
\lambda^{2}+4+\left(\left(1-2 e^{-2 \tau}\right) \lambda+1-4 e^{-2 \tau}\right) e^{-\lambda \tau}=0 .
$$

We notice that $P(j \omega, \tau)+Q(j \omega, \tau)=0$ when $\tau=\frac{1}{2} \ln (2)$ and $\omega=\sqrt{3}$. Therefore Condition (11), which is assumed in [12], is not satisfied. However we can verify that all of our assumptions are satisfied. We have

$F(\omega, \tau)=\omega^{4}-\left(4 e^{-4 \tau}-4 e^{-2 \tau}+9\right) \omega^{2}+12+16 e^{-4 \tau}+8 e^{-2 \tau}$

We find that no $(\omega, \tau) \in \mathbb{R}_{+} \times \mathscr{I}$ simultaneously satisfies (5) and (9), which means $\mathscr{I}^{(1)}=\mathscr{I}$. There are two positive roots of $F_{\tau}(\omega)$ for all $\tau \in \mathscr{I}^{(1)}$, therefore $\omega_{1}^{(1)}(\tau), \omega_{2}^{(1)}(\tau)$ are defined in $\mathscr{I}^{(1)}$. With the corresponding phase functions plotted in the upper diagram of Figure 3, we observe that $\theta_{1}^{(1)}(\tau)$ intersects the horizontal line 0 at $\tau_{1} \approx 0.1982$ and $\theta_{2}^{(1)}(\tau)$ intersects the horizontal line $2 \pi$ at $\tau_{2} \approx 0.6933$. We also have $\omega_{11}=\omega_{1}^{(1)}\left(\tau_{1}\right) \approx 1.4945$ and $\omega_{12}=\omega_{2}^{(1)}\left(\tau_{2}\right) \approx 2.2656$. Computation shows that $\partial_{\omega} F\left(\omega_{11}, \tau_{1}\right)<0$ and $\partial_{\omega} F\left(\omega_{12}, \tau_{2}\right)>0$. From Figure 3 it is easy to see that $\frac{d}{d \tau} \theta_{1}^{(1)}\left(\tau_{1}\right)>0$ and $\frac{d}{d \tau} \theta_{2}^{(1)}\left(\tau_{2}\right)>0$. Accordingly we can deduce that the characteristic root $j \omega_{11}$ moves toward the left-half plane and the characteristic root $j \omega_{12}$ moves towards the right-half plane as $\tau$ increases and goes through $\tau_{1}$ and $\tau_{2}$ respectively. The system has two unstable characteristic roots for $\tau=0$, therefore it is asymptotically stable for $\tau \in\left(\tau_{1}, \tau_{2}\right)$ and unstable for $\tau \in\left[0, \tau_{1}\right) \cup\left(\tau_{2}, 1\right]$.
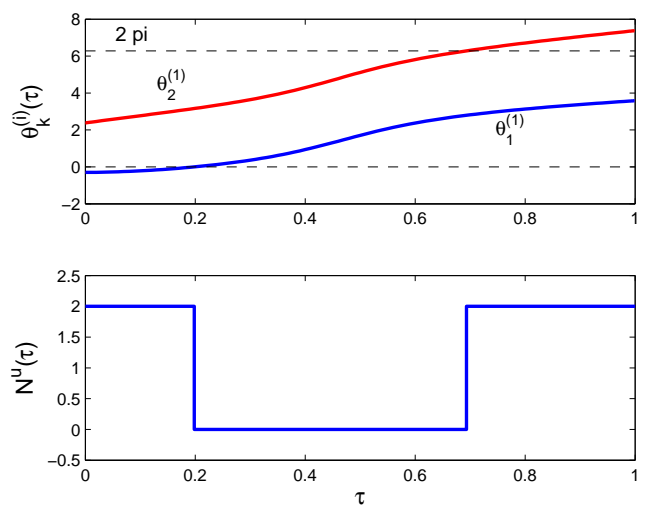

Fig. 3. The stability analysis of Example 3. The graph of $\theta_{1}^{(1)}$ intersects the black-dashed line located at 0 at $\tau_{1}$ and the graph of $\theta_{2}^{(1)}$ intersects the black-dashed line located at $2 \pi$ at $\tau_{2}$. Therefore the system admits imaginary roots at $\tau_{1}$ and $\tau_{2}$. $N^{u}$ is the number of unstable roots of the system.

\section{CONCLUSION}

A method of stability analysis for time-delay systems with coefficients depending on the delay has been developed. The method is an extension of the one given in [12] to a more general case. The method partitions the range of interest for the delay into subintervals so that the magnitude condition yields a fixed number of solutions of frequencies $\omega$ as functions of the delay $\tau$ within each subinterval. The crossing conditions is expressed in a general form, and a simplified derivation for the first order derivative crossing criterion is obtained.

\section{ACKNOWLEDGMENT}

Chi Jin, Islam Boussaada and Silviu Iulian Niculescu are partially financially supported by a grant from Hubert Curien (PHC) BRANCUSI 2017, project number 38390ZL and a grant from Hubert Curien (PHC) BALATON 2018, project number 40502NM.

\section{REFERENCES}

[1] K. L. Cooke and P. van den Driessche. "On zeroes of some transcendental equations,” Funkcialaj Ekvacioj, vol. 29, pp. 77-90, 1986.

[2] F. G. Boese. "Stability with respect to the delay: On a paper by K.L. Cooke and P. van den Driessche," J. Math. Anal. Appl, vol. 228, pp. 293-321, 1998.

[3] K. Gu, V. L. Kharitonov, \& J. Chen (2003). Stability of time-delay systems.

[4] Gu, K. (2012). A review of some subtleties of practical relevance for time-delay systems of neutral type. ISRN Applied Mathematics, Vol 2012, Article ID 725783, 46 pages, doi: 10.5402/2012/725783.

[5] K. Walton and J. E. Marshall, "Direct method for TDS stability analysis," IEE Proc. vol. 134, part D, pp. 101-107, 1987.

[6] L. E. El'Sgol'ts and S. B. Norkin, Introduction to the Theory and Application of Differential Equations with Deviating Arguments, Translated by J. L. Casti, Academic Press, New York, 1973.

[7] E. N. Gryazina, B. T. Polyak, and A. A. Tremba, "D-decomposition technique state-of-the-art," Automation and Remote Control, vol. 69, no. 12, pp. 1991-2026, 2008. 
[8] K. Knopp. Theory of Functions, Parts I and II, Translated to English by F. Bagemihl, Dover, Mineola, NY, 1996.

[9] W. Michiels, \& S. I. Niculescu, (2014). Stability, Control, and Computation for Time-Delay Systems: An Eigenvalue-Based Approach (Vol. 27). Siam.

[10] S. I. Niculescu, (2001). Delay effects on stability: a robust control approach, vol 269. Springer, Heidelberg.

[11] R. Sipahi, S. I. Niculescu, C. T. Abdallah, W. Michiels and K. $\mathrm{Gu}$, "Stability and Stabilization of Systems with Time Delay," in IEEE Control Systems, vol. 31, no. 1, pp. 38-65, Feb. 2011. doi: 10.1109/MCS.2010.939135

[12] E. Beretta, Y. Kuang (2002). Geometric stability switch criteria in delay differential systems with delay dependent parameters. SIAM Journal on Mathematical Analysis, 33(5), 1144-1165.

[13] R. M. Nisbet, W. S. C. Gurney, J. A. J. Metz, Stage structure models applied in evolutionary ecology, Biomathematics, 18 (1989), pp. 428-449.

[14] R. Bence, R. M. Nisbet. Space-limited recruiment in open systems: The importance of time delays, Ecology, 70(1989), pp. 1434-1441.

[15] A. L. Wilmot-Smith, D. Nandy, G. Hornig, "A time delay model for solar and stellar dynamos." The Astrophysical Journal 652.1 (2006): 696

[16] F. Crauste. A review on local asymptotic stability analysis for mathematical models of hematopoietic with delay and delay-dependent coefficients. Annals of the Tiberiu Popoviciu Seminar of functionnal equations, approximation and convexity, 9, 121-143 (2011).

[17] M. S. Lee and C. S. Hsu, "On the $\tau$-decomposition method of stability analysis for retarded dynamical systems," SIAM J. Control, 7:249, 259, 1969.

[18] D. Hertz, E.J. Jury and E. Zeheb, Stability independent and dependent of delay for delay differential systems, J. Franklin Inst. 318(3) (1984) 143-150.

[19] X. G. Li, S. I. Niculescu, A. Cela, et al. Invariance properties for a class of quasipolynomials, Automatica, 2014, 50(3): 890-895.

[20] K. Gu, C. Jin, I. Boussaada and S. I. Niculescu, "Towards more general stability analysis of systems with delay-dependent coefficients," 2016 IEEE 55th Conference on Decision and Control (CDC), Las Vegas, NV, 2016, pp. 3161-3166.

[21] C. Jin, S. -I. Niculescu, I. Boussaada, K. Gu, "Stability analysis of control systems subject to delay-difference feedback," Proceeding of the IFAC World Congress, Toulouse, France (2017).

[22] L.V. Ahlfors. Complex Analysis: An Introduction to the Theory of Analytic Functions of One Complex Variable, L. V. Ahlfors. McGrawHill, 1953.

[23] J. Chen, P. Fu, S. -I. Niculescu, and Z. Guan, "An eigenvalue perturbation approach to stability analysis, part I: eigenvalue series of matrix operators." SIAM Journal on Control and Optimization 48, no. 8 (2010): 5564-5582.

[24] J. Chen, P. Fu, S. -I. Niculescu, and Z. Guan, ”An eigenvalue perturbation approach to stability analysis, part ii: When will zeros of timedelay systems cross imaginary axis?." SIAM Journal on Control and Optimization 48, no. 8 (2010): 5583-5605.

[25] X. G. Li, S. I. Niculescu, A. ela, L. Zhang and X. Li, "A FrequencySweeping Framework for Stability Analysis of Time-Delay Systems," in IEEE Transactions on Automatic Control, vol. 62, no. 8, pp. 3701-3716, Aug. 2017.

[26] D. Israelsson and A. Johnsson, A theory for circumnutations in $\mathrm{He}-$ lianthus annuus. Physiol. Plant. 20, 957-976 (1967).

[27] C. Foley and M. C. Mackey, Mathematical model for G-CSF administration after chemotherapy, Journal of Theoretical Biology, 2009, 257(1): $27-44$.

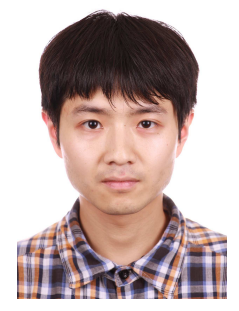

Chi Jin was born in Shanghai, China in 1989. He received the B.S. degree from Tongji University, China, 2012. He is currently a Ph.D student with L2S (Laboratory of Signals and Systems) and Université Paris-Sud located at Gif-sur-Yvette, France. His research interest includes time-delay systems, nonlinear control with applications to automotive vehicles and robotics.

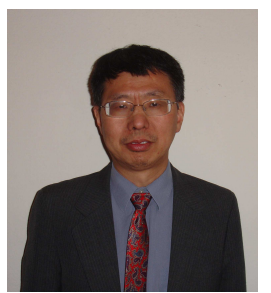

Keqin Gu is a Distinguished Research Professor of the Department of Mechanical and Industrial Engineering, Southern Illinois University Edwardsville. He received B.S. and M.S. from Zhejiang University, and $\mathrm{Ph} . \mathrm{D}$. from Georgia Institute of Technology. His research interest includes control systems and nonlinear dynamical systems, with emphasis on timedelay systems. He authored or co-authored more than 100 papers in archive journals and technical conferences, and is the lead author of the book Stability of Time-Delay Systems. He was the US Coordinator of three US-France cooperative research projects. He is currently serving or served in editorial board of a number of major technical journals in the systems and control area, including Automatica, IEEE Transactions on Automatic Control, and Systems and Control Letters. He also served as a member of the program committee in a number of international conferences and workshops in the area, including Conference on Decision and Control, and American Control Conference.

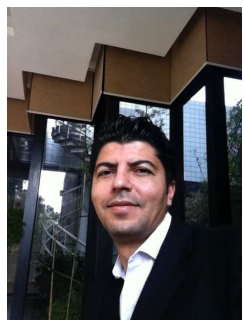

Islam Boussaada received his M.Sc. degree in Mathematics from University Tunis II as well as an M.Sc. degree in Pure Mathematics from University Paris 7 in 2004. In December 2008, he received his Ph.D. degree in Mathematics from Normandy University. In 2016 he obtained the French habilitation (HDR) in Physics from University Paris Saclay. In 2010, I. Boussaada served for two years as a post-doc fellow in control of time-delay systems at L2S Supélec-CNRS-University Paris Sud. Since 2012, he has been serving as an associate professor at IPSA and as an associate researcher at L2S of University Paris Saclay, CentraleSupélec-CNRS-University Paris Sud. Since 2016, he is an associate member of Inria Saclay DISCO project. His research interest belongs to the field of the qualitative theory of dynamical systems and its application in control. It covers the analysis of the delay effect on dynamics, stability and stabilization of delay systems and hyperbolic partial differential equations, oscillations and periodic solutions of functional differential equations, control of vibrations. He authored or co-authored 2 monographs and more than 30 papers in journals, book chapters and international conferences proceedings.

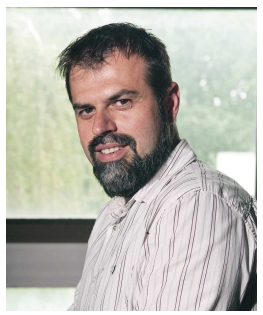

Silviu-Iulian Niculescu received the B.S. degree from the Polytechnical Institute of Bucharest, Romania, the M.Sc., and Ph.D. degrees from the Institut National Polytechnique de Grenoble, France, and the French Habilitation (HDR) from Université de Technologie de Compiègne, all in Automatic Control, in 1992, 1993, 1996, and 2003, respectively. He is currently Research Director at the CNRS(French National Center for Scientific Research) and the head of L2S (Laboratory of Signals and Systems), a joint research unit of CNRS with CentraleSupélec and Université Paris-Sud located at Gif-sur-Yvette. He is author/coauthor of 10 books and of more than 475 scientific papers. His research interests include delay systems, robust control, operator theory, and numerical methods in optimization, and their applications to the design of engineering systems. He is the responsible of the IFAC Research Group on "Time-delay systems" since its creation in October 2007. He served as Associate Editor for several journals in Control area, including the IEEE Transactions on Automatic Control (20032005). Dr. Niculescu was awarded the CNRS Silver and Bronze Medals for scientific research and the Ph.D. Thesis Award from INPG, Grenoble (France) in 2011, 2001 and 1996, respectively. For further information, please visit http://www.12s.centralesupelec.fr/perso/silviu.niculescu. 